\title{
Existence of optimal controls for systems governed by mean-field stochastic differential equations*
}

\author{
Khaled Bahlali $^{\dagger}$, Meriem Mezerdi ${ }^{\ddagger}$ and Brahim Mezerdi $\mathbf{i}^{\ddagger}, *$ \\ ${ }^{\dagger}$ Laboratoire IMATH, Université du Sud-Toulon-Var, B.P 20132, 83957, La Garde Cedex 05, France \\ and CNRS, I2M, Aix Marseille Université, France \\ ${ }^{\ddagger}$ Laboratory of Applied Mathematics, University of Biskra, Po. Box 145, Biskra (07000), Algeria
}

Received 24 July 2014; Accepted 16 October 2014

Copyright (c) 2014, Afrika Statistika. All rights reserved

\begin{abstract}
In this paper, we study the existence of an optimal control for systems, governed by stochastic differential equations of mean-field type. For non linear systems, we prove the existence of an optimal relaxed control, by using tightness techniques and Skorokhod selection theorem. The optimal control is a measure valued process defined on another probability space. In the case where the coefficients are linear maps and the cost functions are convex, we prove by using weak convergence techniques, the existence of an optimal strict control, adapted to the initial filtration.

Résumé. Dans cet article on s'intéresse à l'existence d'un contrôle optimal, pour des systèmes gouvernés par des équations différentielles stochastiques de type champ moyen. Pour les systèmes non linéaires, on démontre un résultat d'existence d'un contrôle optimal relaxé, en utilisant des techniques de tension et le théorème de sélection de Skorokhod. Le contrôle optimal obtenu est un processus à valeurs mesures, défini sur un autre espace de probabilité. Dans le cas où les coefficients sont linéaires et les fonctions de coût sont convexes, on démontre en utilisant des techniques de convergence faible, l'existence d'un contrôle optimal strict, adapté à la filtration initiale.
\end{abstract}

Key words: Mean-field; Stochastic differential equation; Relaxed control; Existence; Tightness; Weak convergence.

AMS 2010 Mathematics Subject Classification : 93E20; 60H30.

\footnotetext{
${ }^{1}$ Corresponding author Brahim Mezerdi: bmezerdi@yahoo.fr

Khaled Bahlali : bahlali@univ-tln.fr

Meriem Mezerdi: m_mezerdi@yahoo.fr

${ }^{*}$ Partially supported by the French-Algerian Cooperation Program, Tassili 13 MDU 887.
} 


\section{Introduction}

The purpose of this paper is to study the problem of existence of an optimal control, for a system governed by a stochastic differential equation of the mean-field type, (MFSDE in short), taking the form:

$$
\left\{\begin{array}{l}
d X_{t}=b\left(t, X_{t}, E\left(\psi\left(X_{t}\right)\right), u_{t}\right) d t+\sigma\left(t, X_{t}, E\left(\Phi\left(X_{t}\right)\right), u_{t}\right) d W_{t} \\
X_{0}=x
\end{array}\right.
$$

$\left(W_{t}, t \geq 0\right)$ is a $d$-dimensional Brownian motion defined on some filtered probability space $\left(\Omega, \mathcal{F},\left(\mathcal{F}_{t}\right), P\right), x$ is the initial state and $u_{t}$ stands for the control variable. $\sigma, b, \psi, \phi$ are deterministic maps.

The expected cost on the time interval $[0, T]$ is of mean field type also and is given by

$$
J(u)=E\left(\int_{0}^{T} h\left(t, X_{t}, E \varphi\left(X_{t}\right), u_{t}\right) d t+g\left(X_{T}, E \lambda\left(X_{T}\right)\right) .\right.
$$

In the state equation and the cost functional, the functions depend not only on the state of the system, but also on the distribution of the state process, via the expectation of some function of the state. MFSDEs are obtained as mean square limits of interacting particle systems of the form:

$$
d X_{t}^{i, n}=b\left(t, X_{t}^{i, n}, 1 / n \sum_{j=1}^{n} \psi\left(X_{t}^{i, n}\right), u_{t}\right) d t+\sigma\left(t, X_{t}^{i, n}, 1 / n \sum_{j=1}^{n} \Phi\left(X_{t}^{i, n}\right), u_{t}\right) d W_{t}
$$

When $n$ goes to infinity, it is proved in Sznitman (1989), in the linear case, that $X_{t}^{i, n}$ converges to $\bar{X}_{t}^{i}$, where all the processes $\bar{X}_{t}^{i}(i=1, \ldots)$, are independent copies of the same process, called the non linear process or the McKean-Vlasov process, which is the unique solution of the MFSDE (1). We refer to Jourdain et al. (2008), to the general case of a non linear dependence of the coefficients upon the process and its distribution and the driving process is a general Lévy process.

Motivated by a recent interest in differential games, control problems where the state process is a MFSDE, where the coefficients depend on the marginal probability law of the solution, have been studied in Ahmed and Ding (2001) and provide interesting models in applications, in particular to game problems Carmona and Delarue (2013); Lasry and Lions (2007). A typical example is the continuous-time Markowitz's mean-variance portfolio selection problem, where one should minimize an objective function involving a quadratic function of the expectation, due to the variance term, see Andersson and Djehiche (2010); Elliott et al. (2013); Yong (2013); Zhou and Li (2000). The main drawback, when dealing with mean field stochastic control problems, is that the state process is not a Markov process and as a consequence, the dynamic programming principle is no longer valid. For this kind of problems, the stochastic maximum principle, provides a powerful tool to solve them, see Andersson and Djehiche (2010); Buckdahn et al. (2011); Chighoub and Mezerdi (2013); Li (2012); Meyer-Brandis et al. (2012); Yang and Tak Kuen (2013); Yong (2013). The SMP gives necessary optimality conditions in terms of the maximization of some hamiltonian and 
K. Bahlali, M. Mezerdi and B. Mezerdi, Afrika Statistika, Vol. 9, 2014, pages 627-645. Existence of optimal controls for systems governed by mean-field stochastic differential equations.

an adjoint process which is the solution of a backward SDE of mean field type, see Buckdahn et al. (2009a,b).

In this paper, we are interested by the existence of an optimal control, where the state equation, as well as the cost function are of mean field type. This kind of result is interesting in itself and particularly when one deals with the stochastic maximum principle. So, it is interesting to know if an optimal control exists and to try to characterize it, by deriving necessary conditions. A control $u^{*}$ is called optimal if it satisfies $J\left(u^{*}\right)=\inf \left\{J(u), u \in \mathcal{U}_{a d}\right\}$, where $\mathcal{U}_{a d}$ is the space of admissible controls, that is measurable, adapted processes with values in some action space $A$. If moreover, $u^{*}$ is in $\mathcal{U}_{a d}$, it is called strict.

For classical control problems, driven by classical SDEs without the mean field part, existence of such a strict optimal control follows from the Filipov-type convexity condition. In the absence of this condition, a strict optimal control may fail to exist. The idea is then to introduce the class of relaxed controls, in which the controller chooses at time $t$, a probability measure $\mu_{t}(d a)$ on the action space $A$, rather than an element $u_{t} \in A$. The set of relaxed controls, when equipped with stable convergence, is a compact separable metrizable space. Note that the class of strict controls could be seen as a subset of the space of relaxed controls, by identifying a strict control $\left(u_{t}\right)$ with the Dirac measure $\delta_{u_{t}}(d a)$. The first existence of an optimal relaxed control has been proved in Fleming (1977), for classical Ito SDEs, where only the drift is controlled. The case of an SDE where the diffusion coefficient depends explicitly on the control variable has been solved in El Karoui et al. (1987); Haussmann (1986), where the optimal relaxed control is shown to be Markovian, see also Haussmann and Lepeltier (1990); Mezerdi and Bahlali (2002); Bahlali et al. (2006). Existence results for systems driven by backward and forward-backward SDEs have been investigated in Bahlali et al. (2010, 2011); Buckdahn et al. (2010).

We establish two main results. We first show the existence of an optimal relaxed control, for control problems driven by non linear MFSDEs. The proof is based on tightness properties of the underlying processes and Skorokhod selection theorem. Our results extend in particular those in El Karoui et al. (1987); Haussmann and Lepeltier (1990); Bahlali et al. (2006), for mean field SDEs. Moreover, due to the compactness of the action space, we show that the relaxed control could be choosen among the so-called sliding controls, which are convex combinations of Dirac measures. As a consequence and under some Fillipov convexity condition, the relaxed control is shown to be strict.The second main result is an existence result for control problems driven by linear MFSDEs. For this particular class of problems, we prove the existence of a strict strong optimal control, that is a control process in $\mathcal{U}_{a d}$, which is adapted to the initial filtration. This means that the admissible controls in this case are adapted to a fixed filtration. The method of proof is based essentially on weak convergence techniques on the space $L_{\mathcal{F}}^{2}$ of square integrable processes and Mazur's theorem on the equality of the strong and weak closure of a convex set. It should be noted that for this case, there is no need to use tightness techniques and to change the initial probability space. The reason is that roughly speaking, in the linear case, the problem reduces to finite dimensional techniques. In particular, our result extends Yong and Zhou (1999), Theorem 5.2 , to mean-field control problems.

Journal home page: www.jafristat.net 
K. Bahlali, M. Mezerdi and B. Mezerdi, Afrika Statistika, Vol. 9, 2014, pages 627-645. Existence of optimal controls for systems governed by mean-field stochastic differential equations.

\section{Existence of optimal relaxed controls for systems driven by non linear MFSDEs}

\subsection{Controlled mean field stochastic differential equations}

Let $\left(W_{t}\right)$ is a $d$-dimensional Brownian motion, defined on a probability space $(\Omega, \mathcal{F}, P)$, endowed with a filtration $\left(\mathcal{F}_{t}\right)$, satisfying the usual conditions. Let $A$ be some compact subset of $\mathbb{R}^{k}$ called the action space or the control set.

We study the existence of optimal controls for systems driven non linear mean field SDEs of the form

$$
\left\{\begin{array}{l}
d X_{t}=b\left(t, X_{t}, E\left(\Psi\left(X_{t}\right)\right), u_{t}\right) d t+\sigma\left(t, X_{t}, E\left(\Phi\left(X_{t}\right)\right) d W_{t}\right. \\
X_{0}=x
\end{array}\right.
$$

and the cost functional over the time interval $[0, T]$ is given by

$$
J(U)=E\left(\int _ { 0 } ^ { T } h \left(t, X_{t}, E\left(\varphi\left(X_{t}\right), u_{t}\right) d t+g\left(X_{T}, E \lambda\left(X_{T}\right)\right)\right.\right.
$$

where $b, \sigma, l, h, g$ and $\psi$ are given functions. The control variable $u_{t}$, is a measurable, $\mathcal{F}_{t}-$ adapted process with values in the action space $A$.

Let us assume the following conditions:

$\left(\mathbf{H}_{\mathbf{1}}\right)$ Assume that

$$
\begin{gathered}
b:[0, T] \times \mathbb{R}^{d} \times \mathbb{R}^{d} \times A \longrightarrow \mathbb{R}^{d} \\
\sigma:[0, T] \times \mathbb{R}^{d} \times \mathbb{R}^{d} \longrightarrow \mathbb{R}^{d} \otimes \mathbb{R}^{d} \\
\Psi: \mathbb{R}^{d} \longrightarrow \mathbb{R}^{d}, \Phi: \mathbb{R}^{d} \longrightarrow \mathbb{R}^{d}
\end{gathered}
$$

are bounded continuous functions and there exists $K>0$ such that for any pairs $\left(x_{1}, y_{1}\right)$ and $\left(x_{2}, y_{2}\right)$ in $\mathbb{R}^{d} \times \mathbb{R}^{d}$ :

$$
\begin{aligned}
& \left|b\left(t, x_{1}, y_{1}, u\right)-b\left(t, x_{2}, y_{2}, u\right)\right| \leq K\left(\left|x_{1}-x_{2}\right|+\left|y_{1}-y_{2}\right|\right) \\
& \left|\sigma\left(t, x_{1}, y_{1}, u\right)-\sigma\left(t, x_{2}, y_{2}, u\right)\right| \leq K\left(\left|x_{1}-x_{2}\right|+\left|y_{1}-y_{2}\right|\right) \\
& \left|\Psi\left(x_{1}\right)-\Psi\left(x_{2}\right)\right| \leq K\left(\left|x_{1}-x_{2}\right|\right) \\
& \left|\Phi\left(x_{1}\right)-\Phi\left(x_{2}\right)\right| \leq K\left(\left|x_{1}-x_{2}\right|\right)
\end{aligned}
$$

$\left(\mathbf{H}_{\mathbf{2}}\right)$ Assume that

$$
\begin{aligned}
& h:[0, T] \times \mathbb{R}^{d} \times \mathbb{R}^{d} \times A \longrightarrow \mathbb{R} \\
& g: \mathbb{R}^{d} \times \mathbb{R}^{d} \longrightarrow \mathbb{R} \\
& \varphi: \mathbb{R}^{d} \longrightarrow \mathbb{R}^{d} \\
& \lambda: \mathbb{R}^{d} \longrightarrow \mathbb{R}^{d}
\end{aligned}
$$

are bounded continuous functions and $h$ is $K$-Lipschiz continuous in the variables $(x, y)$, that is there exists $K>0$ such that for any pairs $\left(x_{1}, y_{1}\right)$ and $\left(x_{2}, y_{2}\right)$ in $\mathbb{R}^{d} \times \mathbb{R}^{d}$ :

$$
\left|h\left(t, x_{1}, y_{1}, u\right)-h\left(t, x_{2}, y_{2}, u\right)\right| \leq K\left(\left|x_{1}-x_{2}\right|+\left|y_{1}-y_{2}\right|\right)
$$

Proposition 1. Under assumption $\left(\mathbf{H}_{\mathbf{1}}\right)$ the MFSDE (3) has a unique strong solution. Moreover for each $p>0$ we have $E\left(\left|X_{t}\right|^{p}\right)<+\infty$.

Journal home page: www.jafristat.net 
Proof. Let us define $\bar{b}(t, x, \mu, a)$ on $[0, T] \times \mathbb{R}^{d} \times \mathbb{M}_{1}\left(\mathbb{R}^{d}\right) \times \mathbb{R}^{k}$ and $\bar{\sigma}(t, x, \mu, a)$ on $[0, T] \times$ $\mathbb{R}^{d} \times \mathbb{M}_{1}\left(\mathbb{R}^{d}\right)$ by

$$
\begin{aligned}
& \bar{b}(t, x, \mu, a)=b\left(., ., \int \Psi(x) d \mu(x), .\right) \\
& \bar{\sigma}(t, x, \mu)=\sigma\left(t, x, \int \Phi(x) d \mu(x)\right)
\end{aligned}
$$

where $\mathbb{M}_{1}\left(\mathbb{R}^{d}\right)$ denotes the space of probability measures in $\mathbb{R}^{d}$.

According to Proposition 1 in Jourdain et al. (2008) it is sufficient to check that $\bar{b}$ and $\bar{\sigma}$ are Lipschitz in $(x, \mu)$. Indeed since the coefficients $b$ and $\sigma$ are Lipschitz continuous in $x$, then $\bar{b}$ and $\bar{\sigma}$ are also Lipschitz in $x$. Moreover one can verify easily that $\bar{b}$ and $\bar{\sigma}$ are also Lipshitz continuous in $\mu$, with respect to the Wasserstein metric

$$
\begin{aligned}
& d(\mu, \nu)=\inf \left\{\left(E^{Q}|X-Y|^{2}\right)^{1 / 2} ; Q \in \mathbb{M}_{1}\left(\mathbb{R}^{d} \times \mathbb{R}^{d}\right), \text { with marginal } \mu, \nu\right\} \\
& =\sup \left\{\int h d(\mu-\nu) ;|h(x)-h(y)| \leq|x-y|\right\}
\end{aligned}
$$

where $\mathbb{M}_{1}\left(\mathbb{R}^{d} \times \mathbb{R}^{d}\right)$ is the space of probability measures on $\mathbb{R}^{d} \times \mathbb{R}^{d}$. Note that the second equality is given by the Kantorovich-Rubinstein theorem Kantorovich and Rubinstein (1958). Since the mappings $b$ and $\Psi$ in the the MFSDE are Lipschitz continuous in $x$ we have

$$
\left|b\left(., ., \int \Psi(x) d \mu(x), .\right)-b\left(., ., \int \Psi(x) d \nu(x), .\right)\right| \leq K\left|\int_{K^{\prime}} \Psi(x) d(\mu(x)-\nu(x))\right|
$$

Similar arguments can be used for $\sigma$.

Using similar techniques as in Proposition 1 in Jourdain et al. (2008), it holds that for each $p>0, E\left(\left|X_{t}\right|^{p}\right)<+\infty$.

\subsection{Relaxed controls}

Our objective is to minimize the cost function, over the class $\mathcal{U}_{a d}$ of admissible controls, that is, adapted processes with values in the set $A$, called the action space. A control $\widehat{u}$ is called optimal if it satisfies $J(\widehat{u})=\inf \left\{J(u), u \in \mathcal{U}_{a d}\right\}$.

If we do not assume convexity conditions, an optimal control may fail to exist in the set $\mathcal{U}_{a d}$ of strict controls even in deterministic control. It should be noted that the set $\mathcal{U}_{a d}$ is not equipped with a compact topology. The idea is then to embed the set of strict controls into a wider class of controls, in which the controller chooses at time $t$, a probability measure $\mu_{t}(d u)$ on the control set $A$, rather than an element $u_{t} \in A$. These measure valued controls are called relaxed controls. It turns out that this class of controls enjoys good topological properties. If $\mu(d u)=\delta_{u_{t}}(d u)$ is a Dirac measure charging $u_{t}$ for each $t$, then we get a strict control as a special case. Thus the set of strict controls may be identified as a subset of of relaxed controls.

Let us consider a simple deterministic example.

Journal home page: www.jafristat.net 
The problem is to minimize the following cost function: $J(u)=\int_{0}^{T}\left(X^{u}(t)\right)^{2} d t$ over the set $\mathcal{U}_{a d}$ of open loop controls, that is, measurable functions $u:[0, T] \rightarrow\{-1,1\}$, where $X^{u}(t)$ denotes the solution of $d X^{u}(t)=u(t) d t, X(0)=0$. We have $\inf _{u \in \mathcal{U}_{a d}} J(u)=0$.

Indeed, consider the following sequence of controls:

$$
u_{n}(t)=(-1)^{k} \text { if } \frac{k T}{n} \leq t \leq \frac{(k+1) T}{n}, 0 \leq k \leq n-1 .
$$

Then clearly $\left|X^{u_{n}}(t)\right| \leq 1 / n$ and $\left|J\left(u_{n}\right)\right| \leq T / n^{2}$ which implies that $\inf _{u \in \mathcal{U}_{a d}} J(u)=0$. There is however no control $\widehat{u}$ such that $J(\widehat{u})=0$. If this would have been the case, then for every $t, X^{\widehat{u}}(t)=0$. This in turn would imply that $u_{t}=0$, which is impossible.

The problem is that the sequence $\left(u_{n}\right)$ has no limit in the space of strict controls. This limit, if it exists, will be the natural candidate for optimality. If we identify $u_{n}(t)$ with the Dirac measure $\delta_{u_{n}(t)}(d u)$, then $\left(\mu_{t}^{n}(d u)\right)_{n}$ converges weakly to $(T / 2) \cdot\left[\delta_{-1}+\delta_{1}\right](d u)$. This suggests that the set of strict controls is too narrow and should be embedded into a wider class with a reacher topological structure for which the control problem becomes solvable. The idea of relaxed control is to replace the $A$-valued process $\left(u_{t}\right)$ with a $\mathbb{M}_{1}(A)$-valued process $\left(\mu_{t}\right)$, where $\mathbb{M}_{1}(A)$ is the space of probability measures equipped with the topology of weak convergence.

In the relaxed form of our control problem we replace in the state equation the process $u_{t}$ by $\mu_{t}$ which a process with values on the space of probability measures on the control set $A$. Then the state process will satisfy, instead of Equation (3), the following equation

$$
\left\{\begin{array}{l}
X_{t}=x+\int_{0}^{t} \int_{A} b\left(s, X_{s}, E\left(\Psi\left(X_{s}\right), a\right) \mu_{s}(d a) \cdot d s+\int_{0}^{t} \sigma\left(s, X_{s}, E\left(\Phi\left(X_{s}\right)\right) d W_{s},\right.\right. \\
X_{0}=x
\end{array}\right.
$$

$\mu_{t}$ is called a relaxed control applied at time $t$. If $\mu_{t}$ is a Dirac measure concentrated at a single point $u_{t}$ then we get a strict control as a particular case of a relaxed control.

\section{The canonical space of the set of relaxed controls}

Let $\mathbb{M}_{1}(A)$ be the space of probability measures on the control set $A$. Let $\mathbb{V}$ be the space of measurable transformations $\mu:[0, T] \longrightarrow \mathbb{M}_{1}(A)$, then $\mu$ can be identified as a nonnegative measure on the product $[0, T] \times A$, by putting for $C \in \mathcal{B}([0, T])$ and $D \in \mathcal{B}(A)$

$$
\bar{\mu}(C \times D)=\int_{C} \mu_{t}(d a) d t
$$

$\bar{\mu}$ can be extended uniquely to an element of $\mathbb{M}_{+}([0, T] \times A)$ the space of Radon measures on $[0, T] \times A$, equipped with the topology of stable convergence. This topology is the weakest topology such that the mapping

$$
\bar{\mu} \longrightarrow \int_{0}^{T} \int_{A} \phi(t, a) \cdot \bar{\mu}(d t, d a)
$$

is continuous for all bounded measurable functions $\phi$ which are continuous in $a$.

Equipped with this topology, $\mathbb{M}_{+}([0, T] \times A)$ is a compact separable metrizable space. Therefore $\mathbb{V}$ as a closed subspace of $\mathbb{M}_{+}([0, T] \times A)$ is also compact (see, El Karoui et al., 1987) for more details. 
Notice that $\mathbb{V}$ can be identified as the space of positive Radon measures on $[0, T] \times A$, whose projections on $[0, T]$ coincide with Lebesgue measure.

Let us define the Borel $\sigma$-field $\overline{\mathbb{V}}$ as the smallest $\sigma$-field such that the mappings

$$
\int_{0}^{T} \int_{A} \phi(t, u) \cdot \mu_{t}(d u) d t
$$

are measurable, where $\phi$ is a bounded measurable function which is continuous in $a$.

Let us also introduce the filtration $\left(\overline{\mathbb{V}}_{t}\right)$ on $\mathbb{V}$, where $\overline{\mathbb{V}}_{t}$ is generated by $\left\{1_{[0, t]} \mu, \mu \in \mathbb{V}\right\}$.

Definition 1. A measure-valued control on the filtered probability space $\left(\Omega, \mathcal{F}, \mathcal{F}_{t}, P\right)$ is a random variable $\mu$ with values in $\mathbb{V}$ such that $\mu(\omega, t, d a)$ is progressively measurable with respect to $\left(\mathcal{F}_{t}\right)$ and such that for each $t, 1_{(0, t]} \cdot \mu$ is $\mathcal{F}_{t}$-measurable.

Now let us introduce the precise definitions of a strict control and relaxed control.

Definition 2. A strict control is a term $\alpha=\left(\Omega, \mathcal{F}, \mathcal{F}_{t}, P, u_{t}, W_{t}, X_{t}\right)$ such that

(1) $\left(\Omega, \mathcal{F}, \mathcal{F}_{t}, P\right)$ is a probability space equipped with a filtration $\left(\mathcal{F}_{t}\right)_{t \geq 0}$ satisfying the usual conditions.

(2) $u_{t}$ is a $A$-valued process, progressively measurable with respect to $\left(\mathcal{F}_{t}\right)$.

(3) $W_{t}$ is a $\left(\mathcal{F}_{t}, P\right)$ - Brownian motion and $\left(W_{t}, X_{t}\right)$ satisfies MFSDE (3).

We denote by $\mathcal{U}_{a d}$ the space of strict controls.

The controls as defined in the last definition are called weak controls, because of the possible change of the probability space and the Brownian motion with $u_{t}$.

Definition 3. A relaxed control is a term $\alpha=\left(\Omega, \mathcal{F}, \mathcal{F}_{t}, P, \mu_{t}, W_{t}, X_{t}\right)$ such that

(1) $\left(\Omega, \mathcal{F}, \mathcal{F}_{t}, P\right)$ is a probability space equipped with a filtration $\left(\mathcal{F}_{t}\right)_{t \geq 0}$ satisfying the usual conditions.

(2) $\mu$ is a measure-valued control on $\left(\Omega, \mathcal{F}, \mathcal{F}_{t}, P\right)$.

(3) $W_{t}$ is a $\left(\mathcal{F}_{t}, P\right)$ - Brownian motion and $\left(W_{t}, X_{t}\right)$ satisfies the following MFSDE (5).

We denote by $\mathcal{R}$ the space of relaxed controls.

Accordingly, the relaxed cost functional will be given by

$$
J(\mu)=E\left(\int _ { 0 } ^ { T } \int _ { A } h \left(t, X_{t}, E\left(\varphi\left(X_{t}\right), a\right) \mu_{t}(d a) d t+g\left(X_{T}, E \lambda\left(X_{T}\right)\right) .\right.\right.
$$

By putting $\widetilde{b}\left(t, X_{t}, E\left(\Psi\left(X_{t}\right), \mu_{t}\right)=\int_{A} b\left(t, X_{t}, E\left(\Psi\left(X_{t}\right), a\right) \mu_{t}(d a)\right.\right.$, it follows that the new drift $\widetilde{b}$ satisfies the same Lipschitz assumptions $\left(\mathbf{H}_{1}\right)$ as $b$. Therefore Equation (5) has a unique solution such that for each $p>0$ we have $E\left(\left|X_{t}\right|^{p}\right)<+\infty$.

Journal home page: www.jafristat.net 


\subsubsection{Approximation of the relaxed model}

By defining the relaxed control problem, a natural question arises on the relation between the strict control problem and the relaxed one. Thanks to the so-called chattering lemma and the continuity of the state process with respect to the control variable, one can prove that the two problems are equivalent. That is the value functions for the two problems are the same. In other words, the infimum of the cost function among strict controls is equal to the infimum of the cost function taken among relaxed controls.

\section{Lemma 1. (Chattering lemma)}

i) Let $\left(\mu_{t}\right)$ be a relaxed control. Then there exists a sequence of adapted processes $\left(u^{n}(t)\right)$ with values in $A$, such that the sequence of random measures $\left(\delta_{u_{t}^{n}}(d a) d t\right)$ converges in $\mathbb{V}$ to $\mu_{t}(d a) d t, P-a . s$.

ii) For any $g$ continuous in $[0, T] \times \mathbb{M}_{1}(A)$ such that $g(t,$.$) is linear, we have P-$ a.s

$$
\lim _{n \rightarrow+\infty} \int_{0}^{t} g\left(s, \delta_{u_{s}^{n}}\right) d s=\int_{0}^{t} g\left(s, \mu_{s}\right) d s \text { uniformly in } t \in[0, T] .
$$

Proof. See El Karoui et al. (1987) and Fleming (1977) Lemma 1 page 152.

Proposition 2. 1) Let $X_{t}, X_{t}^{n}$ be the solutions of state Equation (5) corresponding to $\mu$ and $u^{n}$, where $\mu$ and $u^{n}$ are defined as in the last lemma. Then

$$
\lim _{n \rightarrow \infty} E\left[\sup _{0 \leq t \leq T}\left|X_{t}^{n}-X_{t}\right|^{2}\right]=0 .
$$

2) Let $J\left(u^{n}\right)$ and $J(\mu)$ the expected costs corresponding respectively to $u^{n}$ and $\mu$. Then there exists a subsequence $\left(u^{n_{k}}\right)$ of $\left(u^{n}\right)$ such that $J\left(u^{n_{k}}\right)$ converges to $J(\mu)$.

Proof. 1) Let $\mu$ a relaxed control and $\left(\delta_{u_{t}^{n}}(d a)\right)$ the sequence of atomic measures associated to the sequence of strict controls $\left(u^{n}\right)$, as in the last Lemma. Let $X_{t}, X_{t}^{n}$ the corresponding state processes. Then

$$
\begin{aligned}
\left|X_{t}-X_{t}^{n}\right| & \leq \mid \int_{0}^{t} \int_{A} b\left(s, X_{s}, E\left(\Psi\left(X_{t}\right), u\right) \mu_{s}(d u) \cdot d s-\int_{0}^{t} \int_{A} b\left(s, X_{s}^{n}, E\left(\Psi\left(X_{s}^{n}\right), u\right) \delta_{u_{s}^{n}}(d a) d s \mid\right.\right. \\
& +\mid \int_{0}^{t} \sigma\left(s, X_{s}, E\left(\Phi\left(X_{t}\right)\right) d s-\int_{0}^{t} \sigma\left(s, X_{s}^{n}, E\left(\Phi\left(X_{s}^{n}\right)\right) d s \mid\right.\right. \\
& \leq \mid \int_{0}^{t} \int_{A} b\left(s, X_{s}, E\left(\Psi\left(X_{t}\right), u\right) \mu_{s}(d u) \cdot d s-\int_{0}^{t} \int_{A} b\left(s, X_{s}, E\left(\Psi\left(X_{s}\right), u\right) \delta_{u_{s}^{n}}(d a) d s \mid\right.\right. \\
& +\mid \int_{0}^{t} \int_{A} b\left(s, X_{s}, E\left(\Psi\left(X_{t}\right), u\right) \delta_{u_{s}^{n}}(d a) \cdot d s-\int_{0}^{t} \int_{A} b\left(s, X_{s}^{n}, E\left(\Psi\left(X_{s}^{n}\right), u\right) \delta_{u_{s}^{n}}(d a) d s \mid\right.\right. \\
& +\sup _{s \leq t} \mid \int_{0}^{s} \sigma\left(v, X_{v}, E\left(\Phi\left(X_{v}\right)\right) d W_{v}-\int_{0}^{t} \sigma\left(v, X_{v}^{n}, E\left(\Phi\left(X_{v}^{n}\right)\right) d W_{v} \mid\right.\right.
\end{aligned}
$$

Then by using Burkholder-Davis-Gundy inequality for the martingale part and the fact that all the functions in Equation (5) are Lipschitz continuous, it holds that

$$
E\left(\sup _{0 \leq t \leq T}\left|X_{t}-X_{t}^{n}\right|^{2}\right) \leq K\left[\int_{0}^{T} E\left(\sup _{0 \leq s \leq t}\left|X_{s}-X_{s}^{n}\right|^{2}\right) d t+\varepsilon_{n}\right]
$$


where $K$ is a nonnegative constant and

$$
\begin{aligned}
\varepsilon_{n} & =E\left(\sup _{0 \leq t \leq T} \mid \int_{0}^{t} \int_{A} b\left(s, X_{s}, E\left(\Psi\left(X_{t}\right), u\right) \mu_{s}(d u) \cdot d s\right.\right. \\
& -\int_{0}^{t} \int_{A} b\left(s, X_{s}, E\left(\Psi\left(X_{s}\right), u\right) \delta_{u_{s}^{n}}(d a) d s \mid\right) .
\end{aligned}
$$

By using Lemma 1 ii) and the dominated convergence theorem it holds that $\lim _{n \rightarrow+\infty} \varepsilon_{n}=0$. We conclude by using Gronwall lemma.

2) Property 1) implies that the sequence $\left(X_{t}^{n}\right)$ converges to $X_{t}$ in probability uniformly in $t$, then there exists a subsequence $\left(X_{t}^{n_{k}}\right)$ which converges to $X_{t}, P$-a.s uniformly in $t$. We have

$$
\begin{aligned}
\left|J\left(u^{n_{k}}\right)-J(\mu)\right| \leq E\left[\int_{0}^{T} \int_{A} \mid h\left(t, X_{t}^{n_{k}}, E\left(\varphi\left(X_{t}^{n}\right), a\right)-h\left(t, X_{t}, E\left(\varphi\left(X_{t}\right), a\right) \mid \delta_{u_{t} n_{k}}(d a) d t\right]\right.\right. \\
+E\left[\mid \int_{0}^{T} \int_{A} h\left(t, X_{t}, E\left(\varphi\left(X_{t}\right), a\right) \delta_{u_{t}^{n_{k}}}(d a) d t-\int_{0}^{T} \int_{A} h\left(t, X_{t}, E\left(\varphi\left(X_{t}\right), a\right) \mu_{t}(d a) d t\right]\right]\right. \\
+E\left[\mid g\left(X_{T}^{n_{k}}, E\left(\lambda\left(X_{T}^{n_{k}}\right)\right)-g\left(X_{T}, E\left(\lambda\left(X_{T}\right)\right) \mid\right] .\right.\right.
\end{aligned}
$$

It follows from the continuity and boundness of the functions $h, g, \varphi$ and $\lambda$ with respect to $x$ and $y$, that the first and third terms in the right hand side converge to 0 . The second term in the right hand side tends to 0 by the weak convergence of the sequence $\mu^{n}$ to $\mu$, the continuity and the boundness of $h$ in the variable $a$. We use the dominated convergence theorem to conclude.

Remark 1. As a consequence of Proposition 2, it holds that the value functions for the strict and relaxed control problems are the same.

Notation: In the sequel we denote by: $\mathcal{C}\left([0, T] ; \mathbb{R}^{d}\right)$ : the space of continuous functions from $[0, T]$ into $\mathbb{R}^{d}$, equipped with the topology of uniform convergence.

\subsection{The main result}

The main result of this section is given by the following theorem. Note that this result extends Fleming (1977); El Karoui et al. (1987); Haussmann (1986) to systems driven by mean field SDEs with uncontrolled diffusion coefficient.

Theorem 1. Under assumptions $\left(\mathbf{H}_{\mathbf{1}}\right),\left(\mathbf{H}_{\mathbf{2}}\right)$, the relaxed control problem has an optimal solution.

The proof is based on some auxiliary results related to the tightness of the processes under consideration and the identification of their limits.

Journal home page: www.jafristat.net 
Let $\left(\mu^{n}\right)_{n \geq 0}$ be a minimizing sequence, that is $\lim _{n \rightarrow \infty} J\left(\mu^{n}\right)=\inf _{q \in \mathcal{R}} J(\mu)$ and let $\left(W^{n}, X^{n}\right)$ be the unique solution of our MFSDE:

$$
\left\{\begin{array}{l}
X_{t}^{n}=x+\int_{0}^{t} \int_{A} b\left(s, X_{s}^{n}, E\left(\Psi\left(X_{s}^{n}\right), u\right) \mu_{s}^{n}(d u) \cdot d s+\int_{0}^{t} \sigma\left(s, X_{s}^{n}, E\left(\Phi\left(X_{s}^{n}\right)\right) d W_{s}^{n},\right.\right. \\
X_{0}^{n}=x
\end{array}\right.
$$

The proof of the main result consists in proving that the sequence of distributions of the processes $\left(\mu^{n}, W^{n}, X^{n}\right)$ is tight for a certain topology on the state space and then show that we can extract a subsequence which converges in law to a process $(\widehat{q}, \widehat{W}, \widehat{X})$, which satisfies the same MFSDE. To achieve the proof we show that under some regularity conditions the sequence of cost functionals $\left(J\left(\mu^{n}\right)\right)_{n}$ converges to $J(\widehat{\mu})$ which is equal to $\inf _{\mu \in \mathcal{R}} J(\mu)$ and then $(\widehat{q}, \widehat{W}, \widehat{X})$ is optimal.

Lemma 2. The sequence of distributions of the relaxed controls $\left(\mu^{n}\right)_{n}$ is relatively compact in $\mathbb{V}$.

Proof. The relaxed controls $\mu^{n}$ are random variables on the space $\mathbb{V}$ which is compact. Then by applying Prohorov's theorem yields that the family of distributions associated to $\left(\mu^{n}\right)_{n \geq 0}$ is tight then it is relatively compact.

Lemma 3. Let $\left(W_{t}^{n}, X_{t}^{n}\right)$ be the solution of the MFSDE (7), then the sequence $P_{\left(W^{n}, X^{n}\right)}$ of distributions of $\left(W^{n}, X^{n}\right)$ is relatively compact on the space $\mathcal{C}\left([0, T], \mathbb{R}^{d}\right) \times \mathcal{C}\left([0, T], \mathbb{R}^{d}\right)$, where $\mathcal{C}\left([0, T], \mathbb{R}^{d}\right)$ is endowed with the topology of uniform convergence.

Proof. To prove that the sequence $\left(P_{\left(W^{n}, X^{n}\right)}\right)$ is relatively compact in $\mathcal{C}\left([0, T], \mathbb{R}^{d}\right) \times$ $\mathcal{C}\left([0, T], \mathbb{R}^{d}\right)$ it is sufficient to prove that $\left(P_{W^{n}}\right)$ and $\left(P_{X^{n}}\right)$ are relatively compact in $\mathcal{C}\left([0, T], \mathbb{R}^{d}\right)$. According to Kolmogorov's theorem (Ikeda and Watanabe, 1981, page 18), we need to verify that

a) $\lim _{A \rightarrow+\infty} \inf _{n} P^{n}(\|x(0)\| \leq A)=0$

b) $\lim _{\delta \rightarrow 0} \limsup P^{n}\left(\sup _{\substack{0 \leq s \leq t \leq T \\ t-s<\delta}}\|x(t)-x(s)\| \geq \gamma\right)=0$.

Condition a) is an immediate consequence of the fact that $W^{n}(0)=0$ and $X^{n}(0)=x$.

To prove b) it is sufficient to check that

$$
\begin{aligned}
E\left(\left\|W^{n}(t)-W^{n}(s)\right\|^{4}\right) & \leq C|t-s|^{2} \\
E\left(\left\|X^{n}(t)-X^{n}(s)\right\|^{4}\right) & \leq C|t-s|^{2}
\end{aligned}
$$

for some constants $C_{1}$ and $C_{2}$ independent from $n$.

The first inequality is obvious. Let us verify the second one. We have

$$
\begin{aligned}
E\left(\left\|X_{t}^{n}-X_{s}^{n}\right\|^{4}\right) & \leq M \cdot E\left(\| \int_{s}^{t} \int_{A} b\left(u, X_{u}^{n}, E\left(\Psi\left(X_{u}^{n}\right), a\right) \mu_{s}^{n}(d a) \cdot d s \|^{4}\right.\right. \\
& +\| \int_{s}^{t} \sigma\left(u, X_{u}^{n}, E\left(\Phi\left(X_{u}^{n}\right)\right) d W_{s}^{n} \|^{4}\right)
\end{aligned}
$$

Journal home page: www.jafristat.net 
K. Bahlali, M. Mezerdi and B. Mezerdi, Afrika Statistika, Vol. 9, 2014, pages 627-645. Existence of optimal controls for systems governed by mean-field stochastic differential equations.

where $M$ is some positive constant. Using Burkholder-Davis-Gundy inequality to the martingale part and the fact that $b$ and $\sigma$ are bounded functions yield the desired result.

\section{Proof of Theorem 1}

By using Lemmas 2 and 3, it holds that the sequence of processes $\left(\mu^{n}, W^{n}, X^{n}\right)$ is tight on the space $\mathbb{V} \times \mathcal{C}\left([0, T], \mathbb{R}^{d}\right)^{2}$. Then by the Skorokhod representation theorem, there exists a probability space $(\bar{\Omega}, \overline{\mathcal{F}}, \overline{\mathbb{P}})$, a sequence $\bar{\gamma}^{n}=\left(\bar{\mu}^{n}, \bar{W}^{n}, \bar{X}^{n}\right)$ and $\bar{\gamma}=(\bar{q}, \bar{W}, \bar{X})$ defined on this space such that:

(i) for each $n \in \mathbb{N}$, $\operatorname{law}\left(\gamma^{n}\right)=\operatorname{law}\left(\bar{\gamma}^{n}\right)$,

(ii) there exists a subsequence $\left(\bar{\gamma}^{n_{k}}\right)$ of $\left(\bar{\gamma}^{n}\right)$, still denoted $\left(\bar{\gamma}^{n}\right)$, which converges to $\bar{\gamma}, \overline{\mathbb{P}}$-a.s. on the space $\Gamma$.

This means in particular that the sequence of relaxed controls $\left(\bar{\mu}^{n}\right)$ converges in the stable topology to $\bar{\mu}, \overline{\mathbb{P}}-$ a.s. and $\left(\bar{W}^{n}, \bar{X}^{n}\right)$ converges uniformly to $(\bar{W}, \bar{X}), \overline{\mathbb{P}}-$ a.s.

According to property (i), we get

$$
\left\{\begin{array}{l}
\bar{X}_{t}^{n}=x+\int_{0}^{t} \int_{A} b\left(s, \bar{X}_{s}^{n}, E\left(\Psi\left(\bar{X}_{s}^{n}\right), u\right) \bar{\mu}_{s}^{n}(d u) d s+\int_{0}^{t} \sigma\left(s, \bar{X}_{s}^{n}, E\left(\Phi\left(\bar{X}_{s}^{n}\right)\right) d \bar{W}_{s}^{n},\right.\right. \\
\bar{X}_{0}^{n}=x
\end{array}\right.
$$

The coefficients $b, \sigma, \Psi$ and $\Phi$ being Lipschitz continuous in $(x, y)$, then according to property (ii) and using similar arguments as in Skorokhod (1965) page 32, it holds that

$$
\int_{0}^{t} \int_{A} b\left(s, \bar{X}_{s}^{n}, E\left(\Psi\left(\bar{X}_{s}^{n}\right), u\right) \bar{\mu}_{s}^{n}(d u) d s\right.
$$

converges in probability to

$$
\int_{0}^{t} \int_{K} b\left(s, \bar{X}_{s}, E\left(\Psi\left(\bar{X}_{s}\right), u\right) \bar{\mu}_{s}(d u) d s\right.
$$

and

$$
\int_{0}^{t} \sigma\left(s , \overline { X } _ { s } ^ { n } , E ( \Phi ( \overline { X } _ { s } ^ { n } ) ) d \overline { W } _ { s } ^ { n } \text { converges in probability to } \int _ { 0 } ^ { t } \sigma \left(s, \bar{X}_{s}, E\left(\Phi\left(\bar{X}_{s}\right)\right) d \bar{W}_{s} .\right.\right.
$$

Therefore $\bar{X}$ satisfies the MFSDE

$$
\left\{\begin{array}{l}
\bar{X}_{t}=x+\int_{0}^{t} \int_{K} b\left(s, \bar{X}_{s}, E\left(\Psi\left(\bar{X}_{s}\right), u\right) \bar{\mu}_{s}(d u) d s+\int_{0}^{t} \sigma\left(s, \bar{X}_{s}, E\left(\Phi\left(\bar{X}_{s}\right)\right) d \bar{W}_{s},\right.\right. \\
\bar{X}_{0}^{n}=x .
\end{array}\right.
$$

To finish the proof of Theorem 1 , it remains to verify that $\bar{\mu}$ is an optimal control.

Journal home page: www.jafristat.net 
According to above properties (i)-(ii) and assumption $\left(\mathbf{H}_{2}\right)$, we have

$$
\begin{aligned}
\inf _{\mu \in \mathcal{R}} J(\mu) & =\lim _{n \rightarrow \infty} J\left(\mu^{n}\right) \\
& =\lim _{n \rightarrow \infty} E\left[\int_{0}^{T} \int_{A} h\left(t, X_{t}^{n}, E\left(\varphi\left(X_{t}^{n}\right), a\right) \mu_{t}^{n}(d a) d t+g\left(X_{T}^{n}, E \lambda\left(X_{T}^{n}\right)\right)\right]\right. \\
& =\lim _{n \rightarrow \infty} \bar{E}\left[\int_{0}^{T} \int_{A} h\left(t, \bar{X}_{t}^{n}, E\left(\varphi\left(\bar{X}_{t}^{n}\right), a\right) \bar{\mu}_{t}^{n}(d a) d t+g\left(\bar{X}_{T}^{n}, E \lambda\left(\bar{X}_{T}^{n}\right)\right)\right]\right. \\
& =\bar{E}\left[\int_{0}^{T} \int_{A} h\left(t, \bar{X}_{t}, E\left(\varphi\left(\bar{X}_{t}\right), a\right) \bar{\mu}_{t}(d a) d t+g\left(\bar{X}_{T}, E \lambda\left(\bar{X}_{T}\right)\right)\right] .\right.
\end{aligned}
$$

Hence $\bar{\mu}$ is an optimal control.

The action space $A$ being compact, we prove in the next proposition that the investigation for an optimal relaxed control can be reduced to the so called sliding controls also known as chattering controls. A sliding control is a relaxed control of the form

$$
q_{t}=\sum_{i=1}^{p} \alpha_{i}(t) \delta_{u_{i}(t)}(d a), u_{i}(t) \in A, \alpha_{i}(t) \geq 0 \text { and } \sum_{i=1}^{p} \alpha_{i}(t)=1 .
$$

Proposition 3. Let $\mu$ be a relaxed control and $X$ the corresponding state process. Then one can choose a sliding control

$$
\nu_{t}=\sum_{i=1}^{p} \alpha_{i}(t) \delta_{u_{i}(t)}(d a), u_{i}(t) \in A, \alpha_{i}(t) \geq 0 \text { and } \sum_{i=1}^{p} \alpha_{i}(t)=1
$$

such that

1) $X$ is a solution of the controlled MFSDE

$$
\left\{\begin{aligned}
d X_{t} & =\sum_{i=1}^{p} \alpha_{i}(t) b\left(t, X_{t}, E\left(\Psi\left(X_{t}\right)\right), u_{i}(t)\right) d t+\sigma\left(t, X_{t}, E\left(\Phi\left(X_{t}\right)\right) d W_{t}\right. \\
X_{0} & =x
\end{aligned}\right.
$$

2) $J(\mu)=J(\nu)$.

Proof. Let $\Lambda$ denote the $d+1$-dimensional simplex

$$
\Lambda=\left\{\lambda=\left(\lambda_{0}, \lambda_{1}, \ldots, \lambda_{d+1}\right) ; \lambda_{i} \geq 0 ; \sum_{i=0}^{d+1} \lambda_{i}=1\right\}
$$

and $W$ the $(d+2)$-cartesian product of the set $A$

$$
W=\left\{w=\left(u_{0}, u_{1}, \ldots, u_{d+1}\right) ; u_{i} \in A\right\} .
$$

Journal home page: www.jafristat.net 
Define the function

$$
g(t, \lambda, w)=\sum_{i=0}^{d+1} \lambda_{i} \widetilde{b}\left(t, X_{t}, E\left(\Psi\left(X_{t}\right), u_{i}\right)-\int_{A} \widetilde{b}\left(t, X_{t}, E\left(\Psi\left(X_{t}\right), u\right) \mu_{t}(d u)\right.\right.
$$

where $t \in[0, T], \lambda \in \Lambda, w \in W$ and $\widetilde{b}\left(t, X_{t}, E\left(\Psi\left(X_{t}\right), u_{i}\right)=\left(\begin{array}{c}b\left(t, X_{t}, E\left(\Psi\left(X_{t}\right), u_{i}\right)\right. \\ h\left(t, x_{t}, E\left(\Psi\left(X_{t}\right), u_{i}\right)\right.\end{array}\right)\right.$

Let $\widetilde{b}\left(t, X_{t}, E\left(\Psi\left(X_{t}\right), u_{i}\right), i=0,1, \ldots, d+1\right.$ be the subset of $(d+1)$ arbitrary points in $P\left(t, X_{t}\right)$ where

$$
P\left(t, X_{t}\right)=\left\{\left(b\left(t, X_{t}, E\left(\Psi\left(X_{t}\right), a\right), h\left(t, X_{t}, E\left(\Psi\left(X_{t}\right), a\right)\right) ; a \in A\right\} \subset \mathbb{R}^{d+1}\right.\right.
$$

Then the convex hull of this set is the collection of all points of the form

$$
\sum_{i=0}^{d+1} \lambda_{i} \widetilde{b}\left(t, X_{t}, E\left(\Psi\left(X_{t}\right), u_{i}\right)\right.
$$

If $\mu$ is a relaxed control, then $\int_{A} \widetilde{b}\left(t, X_{t}, E\left(\Psi\left(X_{t}\right), a\right) \mu_{t}(d a) \in \operatorname{Conv}\left(P\left(t, X_{t}\right)\right)\right.$, the convex hull of $P\left(t, X_{t}\right)$. Therefore it follows from Carathéodory's Lemma (which says that the convex hull of a $d$-dimensional set $M$ coincides with the union of the convex hulls of $d+1$ points of $M)$, that for each $(w, t) \in \Omega \times[0, T]$ the equation $g(t, \lambda, w)=0$ admits at least one solution. Moreover the set

$$
\left\{(\omega, \lambda, w) \in \Omega \times \Lambda \times W: \sum_{i=0}^{d+1} \lambda_{i} \widetilde{b}\left(t, X_{t}, E\left(\Psi\left(X_{t}\right), u_{i}\right)=\int_{A} \widetilde{b}\left(t, x_{t}, E\left(\Psi\left(x_{t}\right), a\right) \mu_{t}(d a)\right\}\right.\right.
$$

is measurable with respect to $\mathcal{F}_{t} \otimes \mathcal{B}\left(\mathbb{R}^{d+1}\right) \otimes \mathcal{B}\left(A^{d+1}\right)$ with non empty $\omega$-sections for each $\omega$.

Hence by using a selection theorem El Karoui et al. (1987), there exist measurable $\mathcal{F}_{t}$-adapted processes $\lambda_{t}$ and $w_{t}$ with values, respectively in $\Lambda$ and $W$ such that:

$$
\int_{A} \widetilde{b}\left(t, X_{t}, E\left(\Psi\left(X_{t}\right), a\right) \mu_{t}(d u)=\sum_{i=0}^{d+1} \lambda_{i}(t) \widetilde{b}\left(t, x_{t}, u_{i}(t)\right)\right.
$$

This implies in particular that

$$
\begin{aligned}
\int_{A} b\left(t, X_{t}, E\left(\Psi\left(X_{t}\right), a\right) \mu_{t}(d u)\right. & =\sum_{i=0}^{d+1} \lambda_{i}(t) b\left(t, X_{t}, E\left(\Psi\left(X_{t}\right), u_{i}(t)\right)\right. \\
\int_{A} h\left(t, X_{t}, E\left(\Psi\left(X_{t}\right), a\right) \mu_{t}(d u)\right. & =\sum_{i=0}^{d+1} \lambda_{i}(t) h\left(t, X_{t}, E\left(\Psi\left(X_{t}\right), u_{i}(t)\right)\right.
\end{aligned}
$$

which ends the proof.

The next corollary is important in applications. It says that under the so-called Fillipov condition an optimal strict control exists.

Journal home page: www.jafristat.net 
Corollary 1. Assume that the set

$$
P\left(t, X_{t}\right)=\left\{\left(b\left(t, X_{t}, E\left(\Psi\left(X_{t}\right), a\right), h\left(t, X_{t}, E\left(\Psi\left(X_{t}\right), a\right)\right) ; a \in A\right\} \subset \mathbb{R}^{d+1}\right.\right.
$$

is convex. Then the relaxed optimal control is realized by a strict control.

Proof. The proof is a direct consequence of Proposition 3. Indeed by mimicking the proof of Proposition 3, it follows that for each relaxed control $\mu$ we have

$$
\int_{A} \widetilde{b}\left(t, X_{t}, E\left(\Psi\left(X_{t}\right), a\right) \mu_{t}(d a) \in \operatorname{Conv}\left(P\left(t, X_{t}\right)\right) .\right.
$$

Since $P\left(t, X_{t}\right)$ is convex then $\operatorname{Conv}\left(P\left(t, X_{t}\right)\right)=P\left(t, X_{t}\right)$.

Then applying the same arguments, there exists a measurable $\mathcal{F}_{t}$-adapted process $u(t)$ such that

$$
\begin{aligned}
& \int_{A} b\left(t, X_{t}, E\left(\Psi\left(X_{t}\right), a\right) \mu_{t}(d u)=b\left(t, X_{t}, u(t)\right),\right. \\
& \int_{A} h\left(t, X_{t}, E\left(\Psi\left(X_{t}\right), a\right) \mu_{t}(d u)=h\left(t, X_{t}, u(t)\right)\right.
\end{aligned}
$$

which implies that $X_{t}$ is a solution of the MFSDE

$$
\left\{\begin{array}{l}
d X_{t}=b\left(t, X_{t}, E\left(\Psi\left(X_{t}\right)\right), u(t)\right) d t+\sigma\left(t, X_{t}, E\left(\Phi\left(X_{t}\right)\right) d W_{t}\right. \\
X_{0}=x
\end{array}\right.
$$

and $J(\mu)=J(u)$. This ends the proof.

\section{Existence of an optimal strong control for linear SDEs}

\subsection{Formulation of the problem}

In this section, we assume that the coefficients of our mean field SDE are linear, while the running and final costs remain non linear. Moreover we assume convexity of the instantaneous and terminal cost functions, as well as the action space $A$. We prove the existence of an optimal strong control, that is a control which is adapted to the initial filtration. Note that for this kind of problems there is no need to use tightness techniques and Skorokhod selection theorem. The techniques used are based on weak convergence techniques in $L_{\mathcal{F}}^{2}\left(0, T, \mathbb{R}^{k}\right)$ and Mazur's theorem.

Definition 4. Let $A$ be a subset in $\mathbb{R}^{d}$ called the action space. An admissible control is a measurable, $\mathcal{F}_{t}$-adapted process with values on the action space $A$ such that:

$$
E\left[\int_{0}^{T}\left|u_{t}\right|^{2} d t\right]<+\infty .
$$

Let us denote $\mathcal{U}_{a d}$ the space of all admissible controls which can be written as:

$$
\mathcal{U}_{a d} \triangleq\left\{u \in L_{\mathcal{F}}^{2}\left(0, T, \mathbb{R}^{d}\right) / u(t) \in A, d t-\text { a.e. } P \text {-a.s }\right\},
$$

where $L_{\mathcal{F}}^{2}\left(0, T, \mathbb{R}^{d}\right)$ is the space of measurable $\mathcal{F}_{t}$-adapted processes with values in $\mathbb{R}^{d}$. 
Assume that for any ablissible control $u$, the state of our system is driven by the following linear MFSDE

$$
\left\{\begin{array}{l}
d X_{t}=\left(A \cdot X_{t} d t+B \cdot E\left(X_{t}\right)+C \cdot u(t)\right) d t+\left(A_{1} \cdot X_{t}+B_{1} \cdot E\left(X_{t}\right)+C_{1} \cdot u(t)\right) d W_{t} \\
X_{0}=x_{0}
\end{array}\right.
$$

where:

- $A, B, A_{1}, B_{1}$ are $d \times d$ matrices.

$-C, C_{1}$ are $d \times d$ matrices.

The cost functional is given as follows:

$$
J(u)=E\left[\int_{0}^{T} h\left(t, X_{t}, E\left(X_{t}\right), u_{t}\right) d t+g\left(X_{T}, E\left(X_{T}\right)\right)\right]
$$

where

$$
\begin{gathered}
h:[0, T] \times \mathbb{R}^{d} \times \mathbb{R}^{d} \times A \rightarrow \mathbb{R} \\
g: \mathbb{R}^{d} \times \mathbb{R}^{d} \rightarrow \mathbb{R} .
\end{gathered}
$$

Let us assume the following.

$\left(\mathbf{H}_{3}\right)$ The set $A \subset \mathbb{R}^{d}$ is convex and closed and the functions $h$ and $g$ are convex and for some $\delta, k>0$

$$
h(t, x, y, u) \geq \delta\left|u^{2}\right|-k, g(x, y) \geq-k, \text { for every }(t, x, y, u) \in[0, T] \times \mathbb{R}^{d} \times \mathbb{R}^{d} \times A
$$

$\left(\mathbf{H}_{4}\right)$ The set $A \subset \mathbb{R}^{d}$ is convex and compact and the functions $h$ and $g$ are convex.

Lemma 4. For every admissible control u, Equation (8) admits a unique strong solution such that for any $p \geq 1$

$$
E\left[\sup _{0 \leq t \leq T}\left|X_{t}\right|^{p}\right]<+\infty
$$

Proof. The coefficients of Equation (8) are linear mappings, then they are globally Lipschitz on the state variable. Then using similar technqiues as in Proposition 1, they are also Lipschitz on the marginal distribution of the state process $X$. Then applying Jourdain et al. (2008), Proposition 1, it holds that Equation (8) has a unique strong solution, such that for any $p \geq 1, E\left(\sup _{0 \leq t \leq T}\left|X_{t}\right|^{p}\right)<+\infty$.

\subsubsection{Existence of an optimal control}

The following theorem could be seen as a generalization of Yong and Zhou (1999), Theorem 5.2 , to systems driven by mean-field stochatic differential equations.

Theorem 2. Under $\left(\boldsymbol{H}_{\mathbf{3}}\right)$ or $\left(\boldsymbol{H}_{\mathbf{4}}\right)$, if the control problem is finite, then it admits an optimal control. 
Proof. Suppose that the control problem is finite ie: $\inf \left\{J(u), u \in \mathcal{U}_{a d}\right\}<+\infty$. In particular, this assumption is fulfilled if the running cost $h(t, x, y, u)$ and the final cost $g(x, y)$ have linear growth with respect to $(x, y)$.

Then $\forall \varepsilon>0, \exists u_{\varepsilon} \in \mathcal{U}_{a d}, J\left(u_{\varepsilon}\right) \leq \inf \left\{J(u), u \in \mathcal{U}_{a d}\right\}+\varepsilon$.

If we take $\varepsilon_{j}=1 / 2^{j}$, then $\exists u^{j} \in \mathcal{U}_{a d}, J\left(u^{j}\right) \leq \inf \left\{J(u), u \in \mathcal{U}_{a d}\right\}+1 / 2^{j}$.

It is clear that $\lim _{j \rightarrow+\infty} J\left(u^{j}\right)=\inf \left\{J(u), u \in \mathcal{U}_{a d}\right\}$ and then a minimizing sequence exists.

By using assumption $\left(\mathbf{H}_{\mathbf{1}}\right)$ we obtain:

$\left.J\left(u^{j}\right)=E\left[\int_{0}^{T} h\left(t, X_{t}^{j}, E\left(X_{t}^{j}\right), u_{t}^{j}\right) d t+g\left(X_{T}^{j}, E\left(X_{T}^{j}\right)\right)\right] \geq E\left[\int_{0}^{T}\left(\delta\left|u^{j}(t)\right|^{2}-K\right) d t-K\right)\right]$.

Then for each $j \geq 1$

$$
E \int_{0}^{T}\left|u^{j}(t)\right|^{2} d t \leq K T+K+J\left(u^{j}\right)
$$

Since the sequence $\left(J\left(u^{j}\right)\right)$ is convergent, then $\sup _{j}\left|J\left(u^{j}\right)\right| \leq C$. This implies that

$$
E\left[\int_{0}^{T}\left|u^{j}(t)\right|^{2} d t\right]=\left\|u^{j}\right\|_{L_{\mathcal{F}}^{2}}^{2}<K T+K+C .
$$

Therefore, the sequence of admissible controls $\left(u^{j}\right)$ is uniformly bounded in the space $L_{\mathcal{F}}^{2}\left(0, T, \mathbb{R}^{k}\right)$.

Alternatively, if we assume $\left(\mathbf{H}_{4}\right)$, then the set $A$ is compact in $\mathbb{R}^{d}$ and the sequence $\left(u^{j}\right)$ is bounded in $L_{\mathcal{F}}^{2}$. Then assuming either $\left(\mathbf{H}_{\mathbf{3}}\right)$ or $\left(\mathbf{H}_{4}\right)$, the sequence $\left(u^{j}\right)$ is bounded in $L_{\mathcal{F}}^{2}$. This implies that under assumption $\left(\mathbf{H}_{3}\right)$ or $\left(\mathbf{H}_{4}\right),\left(u^{j}\right)$ is relatively compact in $L_{\mathcal{F}}^{2}$ equipped with the weak topology. Then there exists a subsequence, still denoted by $\left(u^{j}\right)$ and a process $\bar{u}$, such that $\left(u^{j}\right)$ converges weakly in $L_{\mathcal{F}}^{2}$ to $\bar{u}$.

By Mazur's theorem (Yosida, 1980, Theorem 2 page 120), there exists a sequence of convex combinations of $\left(u^{j}\right)$ which converges strongly to $\bar{u}$.

This means that there exist real numbers $\left(\alpha_{i j}\right)$, with $\alpha_{i j} \geq 0, \sum_{i>1} \alpha_{i j}=1, \alpha_{i j}$ are equal to zero except for a finite number, such that if we denote $\widetilde{u}^{j}=\sum_{i \geq 1} \alpha_{i j} \cdot u_{i+j}$, then $\widetilde{u}^{j} \rightarrow \bar{u}$ strongly in $L_{\mathcal{F}}^{2}$.

Since $A \subseteq \mathbb{R}^{d}$ is convex and closed, then $\bar{u}$ is an admissible control belonging to $\mathcal{U}_{a d}$.

Let us denote by $\widetilde{X}^{j}$ (resp. $\bar{X}$ ) the solution of the state Equation (8), associated to the admissible control $\widetilde{u}^{j}$ (resp. $\bar{u}$ ). Then, by using classical arguments, from stochastic calculus, one can prove that

$$
\tilde{X}^{j} \longrightarrow \bar{X} \text { strongly in } C_{\mathcal{F}}\left([0, T], \mathbb{R}^{n}\right)
$$

Journal home page: www.jafristat.net 
where

$$
\begin{aligned}
C_{\mathcal{F}}\left([0, T], \mathbb{R}^{n}\right) & =\left\{X: \Omega \times[0, T] \rightarrow \mathbb{R}^{n}, \mathcal{F}_{t}\right. \text {-adapted, } \\
& \text { continuous such that: } \left.E\left(\sup _{0 \leq t \leq T}\left|X_{t}\right|\right)<+\infty\right\} .
\end{aligned}
$$

Let us give the outlines of the proof.

$$
\begin{aligned}
\left(\widetilde{X}_{t}^{j}-\bar{X}_{t}\right) & =\int_{0}^{t}\left(\left(A\left(\widetilde{X}_{s}^{j}-\bar{X}_{s}\right)+B\left(E\left(\widetilde{X}_{s}^{j}\right)-E\left(\bar{X}_{s}\right)\right)+C\left(\widetilde{u}_{s}^{j}-\bar{u}_{s}\right)\right) d s\right. \\
& +\int_{0}^{t}\left(A_{1}\left(\widetilde{X}_{s}^{j}-\bar{X}_{s}\right)+B_{1}\left(E\left(\widetilde{X}_{s}^{j}\right)-E\left(\bar{X}_{s}\right)\right)+C_{1}\left(\widetilde{u}_{s}^{j}-\bar{u}_{s}\right) d W_{s} .\right.
\end{aligned}
$$

Then

$$
\begin{aligned}
\left(\sup _{s \leq t}\left|\tilde{X}_{s}^{j}-\bar{X}_{s}\right|\right)^{2} & \leq \int_{0}^{t}\|A\|^{2}\left(\sup _{0 \leq v \leq s} \mid\left(\widetilde{X}_{v}^{j}-\bar{X}_{v} \mid\right)^{2}+\|B\|^{2}\left(\sup _{0 \leq v \leq s}\left|E\left(\tilde{X}_{v}^{j}\right)-E\left(\bar{X}_{v}\right)\right|\right)^{2} d t\right. \\
& +\int_{0}^{t}\|C\|^{2}\left|\widetilde{u}_{v}^{j}-\bar{u}_{v}\right|^{2} d t \\
& +\sup \left(\left|\int_{0}^{t} A_{1}\left(\tilde{X}_{s}^{j}-\bar{X}_{s}\right)+B_{1}\left(E\left(\tilde{X}_{s}^{j}\right)-E\left(\bar{X}_{s}\right)\right)+C_{1}\left(\widetilde{u}_{s}^{j}-\bar{u}_{s}\right) d W\right|\right)^{2} .
\end{aligned}
$$

By applying the Burkholder-Davis-Gundy inequality to the martingale part, we obtain:

$$
E\left[\sup _{s \leq T}\left|\widetilde{X}_{s}^{j}-\bar{X}_{s}\right|^{2}\right] \leq C_{1} \int_{0}^{t} E\left(\sup _{u \leq s}\left|\widetilde{X}_{u}^{j}-\bar{X}_{u}\right|^{2}\right) d s+C_{2} E \int_{0}^{t}\left|\widetilde{u}_{s}^{j}-\bar{u}_{s}\right|^{2} d s .
$$

If we set $f(t)=E\left[\sup _{s \leq t}\left|\tilde{X}_{s}^{j}-\bar{X}_{s}\right|^{2}\right]$, then

$$
f(t) \leq C_{1} \int_{0}^{t} f(s) d s+C_{2} E \int_{0}^{t}\left|\widetilde{u}_{s}^{j}-\bar{u}_{s}\right|^{2} d s
$$

By applying Gronwall's lemma, there exists a positive constant $C$ such that:

$$
E\left[\sup _{s \leq T}\left|\widetilde{X}_{s}^{j}-\bar{X}_{s}\right|^{2}\right] \leq C \cdot E \int_{0}^{t}\left|\widetilde{u}_{s}^{j}-\bar{u}_{s}\right|^{2} d s
$$


Since $\left(\widetilde{u}^{j}\right)$ converges to $\bar{u}$ strongly in $L_{\mathcal{F}}^{2}$, that is $\lim _{j \rightarrow \infty} E \int_{0}^{t}\left|\widetilde{u}_{s}^{j}-\bar{u}_{s}\right|^{2}=0$, we conclude that

$$
\lim _{j \rightarrow \infty} E\left[\sup _{s \leq T}\left|\tilde{X}_{s}^{j}-\bar{X}_{s}\right|^{2}\right]=0
$$

Now, let us verify that $\bar{u}$ is an optimal control. The continuity and the convexity of the cost functions $h$ and $g$ imply that

$$
\begin{aligned}
J(\bar{u}) & =\lim _{j \rightarrow \infty} J\left(\widetilde{u}^{j}\right) \leq \lim _{j \rightarrow \infty} \sum_{i \geq 1} \alpha_{i j} . J\left(u^{i+j}\right) \\
& \leq \lim _{j \rightarrow \infty} \sum_{i \geq 1}^{i_{j}} \alpha_{i j} . \max \left\{J\left(u^{i+j}\right), i=1, \ldots, i_{j}\right\} \\
& =\lim _{j \rightarrow \infty} \sum_{i=1}^{i_{j}} \alpha_{i j} . J\left(u^{j_{0}+j}\right) \\
& =\lim _{j \rightarrow \infty} J\left(u^{j_{0}+j}\right)=\inf _{u \in \mathcal{U}_{a d}} J(u)
\end{aligned}
$$

because $\left(u^{j}\right)$ is a minimizing sequence. The proof is now completed.

\section{References}

Ahmed, N.U. and Ding, X. 2001. Controlled McKean-Vlasov equations. Comm. in Appl. Analysis. 5(2), 183-206.

Andersson, D. and Djehiche, B. 2010. A maximum principle for SDEs of mean-field type. Appl. Math. and Optim. 63(3), 341-356.

Bahlali, K., Gherbal, B. and Mezerdi, B. 2010. Existence and optimality conditions in stochastic control of linear BSDEs, Rand. Oper. Stoch. Equ. 18(3), 185-197.

Bahlali, K., Gherbal, B. and Mezerdi, B. 2011. Existence of optimal controls for systems driven by FBSDEs, Systems and Cont. Lett. 60, 344-349.

Bahlali, S. Djehiche, B. and Mezerdi, B. 2006. Approximation and optimality necessary conditions in relaxed stochastic control problems. J. Appl. Math. Stoch. Anal. Article ID $72762,1-23$

Buckdahn, R. Djehiche, B. and Li, J. 2011. A general stochastic maximum principle for SDEs of mean-field type. Appl. Math. Optim. 64(2), 197-216.

Buckdahn, R. Djehiche, B. Li, J. and Peng, S. 2009. Mean-Field backward stochastic differential equations: a limit approach. Ann. Prob., 37(4), 1524-1565.

Buckdahn, R. Labed, B. Rainer, C. Tamer, L. 2010. Existence of an optimal control for stochastic systems with nonlinear cost functional, Stochastics. 82(3), 241-256.

Buckdahn, R. Li, J. and Peng, S. 2009. Mean-Field backward stochastic differential equations and related partial diferential equations. Stoch. Proc. and their Appl. 119, 3133-3154.

Carmona, R. Delarue, F. 2013. Probabilistic analysis of mean-field games. SIAM J. Control Optim. 51(4), 2705-2734.

Chighoub, F. Mezerdi, B. 2013. A stochastic maximum principle in mean-field optimal control problems for jump diffusions. Arab J. Math. Sci. 19(2), 223-241. 
Elliott, R. Li, X. and Ni, Y.H. 2013. Discrete time mean-field stochastic linear-quadratic optimal control problems. Automatica J. IFAC. 49(11), 3222-3233.

El Karoui, N. Nguyen, D. H. and Jeanblanc-Picqué, M. 1987. Compactification methods in the control of degenerate diffusions: existence of an optimal control, Stochastics. 20(3), 169-219.

Fleming, W.H. 1977. Generalized solutions in optimal stochastic control, Differential Games and Control theory II, Proceedings of 2nd Conference, University of Rhode Island, Kingston, RI, 1976, Lecture Notes in Pure and Appl. Math. 30, Marcel Dekker, New York. 147-165.

Haussmann, U.G. 1986. Existence of optimal Markovian controls for degenerate diffusions, Stochastic Differential Systems, Lecture Notes in Control and Inform. Sci. 78, Springer, Berlin. 171-186.

Haussmann U.G. and Lepeltier, J.-P. 1990. On the existence of optimal controls. SIAM J. Cont. Optim. 28(4), 851-902.

Ikeda N. and Watanabe, S. 1981. Stochastic Differential Equations and Diffusion Processes, North-Holland Mathematical Library, Vol. 24, North-Holland, Amsterdam.

Jourdain, B. Méléard, S. Woyczynski, W. 2008. Nonlinear SDEs driven by Lévy processes and related PDEs. Alea 4, 1-29.

Kantorovich, L. B. and Rubinstein, G.S. 1958. On the space of completely additive functions. Vestn. Leningr. Univ., Mat. Meh. Astron. 13(7), 52-59.

Lasry, J.M. and Lions, P.L. 2007. Mean-field games. Japan. J. Math. 2, 229-260.

Li, J. 2012. Stochastic maximum principle in the mean-field controls. Automatica. 48(2), 366-373.

Meyer-Brandis, T. Øksendal, B. and Zhou, X.Y. 2012. A mean-field stochastic maximum principle via Malliavin calculus. Stochastics. 84(5-6), 643-666.

Mezerdi, B. and Bahlali, S. 2002. Necessary conditions for optimality in relaxed stochastic control problems. Stochastics and Stoch. Reports. 73(3-4), 201-218.

Skorokhod, A.V. 1965. Studies in the theory of random processes. Adisson-Wesley, originally published in Kiev 1961.

Sznitman, A.S. 1989. Topics in propagation of chaos. In Ecole de Probabilités de Saint Flour, XIX-1989. Lecture Notes in Math. Springer, Berlin. 1464, 165-251.

Yang, S. Tak Kuen, S. 2013. The maximum principle for a jump-diffusion mean-field model and its application to the mean-variance problem. Nonlinear Anal. Theory, Meth. and Appl. 86, 58-73.

Yong, J. 2013. Linear-quadratic optimal control problems for mean-field stochastic differential equations. SIAM J. Control Optim. 51(4), 2809-2838.

Yong J. and Zhou, X.Y 1999. Stochastic controls, Hamiltonian Systems and HJB Equations. Springer, New York.

Yosida, K., 1980. Functional analysis, 6th edition, Springer-Verlag, Berlin.

Zhou, X.Y. and Li, D. 2000. Continuous-Time Mean-Variance Portfolio Selection: A Stochastic LQ Framework. Appl. Math. Optim. 42, 19-33. 